(Aus der Privatheilanstalt für Frauenkrankheiten und Geburtshülfe von Prof. Dr. Dührssen-Berlin.)

\title{
Darf die Bossi'sche Methode dem praktischen Arzt empfohlen werden?
}

(Nebst Mittheilung zweier weiteren Fälle von vaginalem Kaisersehnitt und kritischer Betrachtung seiner Concurrenzmethoden.)

Von

A. Dührssen.

Die vorstehende Frage ist trotz des Einspruchs einer Reihe von Autoren von Leopold in bejahendem Sinn entschieden worden. Auch in der jüngsten Arbeit aus seiner Klinik hebt Ehrlich ${ }^{1}$ ) ein Wort von Bossi hervor, welcher den hohen Werth seines Verfahrens für den practischen Arzt darin sieht, dass es ihm in allen, wenn auch noch so verzweifelten Lagen eine sichere und gefahrlose Methode zur Einleitung der Geburt zur Verfügung stellt, welche in der ärmlichsten Dachkammer und ohne geschulte Assistenz zur Anwendung gelangen könne - und dass es nicht nur bei Erstgebärenden mit unverkürztem Collum mit Erfolg anwendbar ist, sondern auch in einer ausserordentlich kurzen Zeit zum Ziel (d. h. zur Entwickelung des Kindes) führt, ohne dass sich schwierige Nähte nöthig machen. Auch beherrscht nach Bossi der Operateur die Situation, indem er mit Sicherheit den Uterus entleeren könne, und zwar in derjenigen Zeit, die er dafür angebracht halte.

Aus dem ganzen Tenor der Arbeit von Ehrlich geht hervor, dass Leopold auch jetzt noch die Bossi'sche Anschaung vertritt, dass seine Methode für den Practiker zu empfehlen sei.

Gegen diese Anschauung habe ich schwere Bedenken. Die Mittheilungen über die Bossi'sche Methode beweisen, dass dieselbe

1) Dieses Archiv. Bd. 73. H. 3. 
keine gefahrlose Methode, dass sie auch keine sichere, d. h. in allen Fällen die Extraction eines unzerstückelten Kindes ormöglichende Methode ist, da sie bei erhaltenem Collum versagen kann dass sie unter Umständen sehr zeitraubend ist, da ihre Ausführung eine Dauer bis zu 3 Stunden beanspruchen kann - dass sie schwierige Nähte erforderlich machen kann, und dass infolgedessen der Operateur bei der Bossi'schen Methode die Situation durchaus nicht beherrscht, sondern auf's Ungewisse ohne jede Ahnung, ob er durch die Methode ohne Gefahr für die Mutter ein lebensfrisches Kind wird entwickeln können, darauf losschrauben muss.

Berücksichtigt man dabei noch, dass der Geburtshelfer während der ganzen Dauer der Dilatation, also bis zu 3 Stunden, die Finger am Muttermund halten muss, so muss man zugeben, dass die Methode nicht nur für den Geburtshelfer, sondern auch ganz besonders für die Kreissende höchst lästig, für letztere anch gefährlich ist, da die lange Dauer des Fingercontacts mit der Cervix eine Infection begünstigt. Ein weiteres gefährliches Moment für dio Kreissende liegt in der langen Daver der Narkose, die bei Eklampsie nicht zu entbehren ist. Schon aus diesen Ueberlegungen ergiebt sich, dass die Bossi'sehe Methode dem neito, tuto et jueunde" nicht Rechnung trägt.

Ganz besondere Bedenken habe ich auch gegen die Empfehlung der hohen Zange, welche sich ja an die Dilatation nach Bossi anschliessen soll. Die maassgebenden geburtshülflichen Lehrbücher können den Practiker nicht genug vor der Anwendung der hohen Zange warnen - und hier wird sie im Anschluss an ein bedenkliches Dilatationsverfahren als die schulgerechte entbindende Operation hingestellt. Und warum hat die hohe Zange hier den Vorzug vor der Wendung? Weil eben in vielen Fällen die Bossi'sche Methode die Cervix nur mangelhaft erweitert, und dann letztere den nachfolgenden Kopf nicht passiren lässt. Die hohe Zange ist hier eben ein nothwendiges Uebel: Der gewaltsame Zangenzug muss die Erweiterung herbeifühen, welche der Dilatator nicht zu erzielen vermochte. Gelingt die Extraction, so trägt das Kind sicherlich in Folge des starken Zangendrucks oft einen dauernden Schaden davon, oder es entstehen Cervixrisse, welche voll und ganz das Conto der Bossi'schen Methode belasten. Denn wenn sie auch nicht durch die Dilatation entstanden sind, so sind sie deswegen entstanden, weil die Dilatation keine genügende war. Yon einer Dilatationsmethode aber, die als ein so vorzüg- 
liches Verfahren gerühmt wird, muss man eine solche Erweiterung der wcichen Geburtswege verlangen, dass sie der nachfolgenden Entwickelung des Kindes nicht den geringsten Widerstand entgegensetzen. Bleiben noch Widerstände zu überwinden, dann gelingt ferner die Extraction des Kindes vielfach nicht, und es muss dann so häufig zu der Perforation gegriffen werden, wie das z. B. bei den von Ehrlich berichteten Fällen sich ereignete: Auch diese vernichteten kindlichen Leben belasten das Conto der Bossi'schen Methode!

Ich bin mit vielen anderen Autoren derAnsicht, dass, wenn überhaupt, heutzutage nur der specialistisch ausgebildete Geburtsheller die Bossi'sche Methode anwonden darf. Wird dieser Satz aber anerkannt, dann ist nicht zu verstehen, warum nicht der vaginale Kaiserschnitt die Bossi'sche Methode völlig verdrängen sollte. Denn der vaginale Kaiserschnitt erfordert zu seiner Ausführung nur einen modernen Gynäkologen im Uebrigen ist er überall dort anwendbar, wo die Bossi'sche Methode zum Ziele führen soll, aber es häufig nicht thut, und hat alle die Vorzüge, welche der Bossi'schen Methode vielfach zu Unrecht nachgerühmt werden, und nicht die Nachtheile, welche bei der Bossi'schen Methode doch thatsächlich oft beobachtet sind. Ist $n a ̈ m l i c h$ bei mangelhaft erweiterter oder geschlossener Cervix eine Lebensgefahr für die Mutter oder das Kind vorhanden, welche durch die sofortige Entwickelung des Kindes beseitigt oder gemildert wird, so ist der vaginale Kaiserschnitt die einzige Methode, welche auf vaginalem Wege binnen weniger Minuten (5-10) die Extraction eines lebensfrischen Kindes ohne Gefährdung der Mutter mit Sicherheit ermöglicht - vorausgesetzt, dass der Operateur ein moderner Gynäkologe ist, dass er an den Kaiserschnitt nicht die Zange, sondern die Wendung und Extraction anschliesst und dass er bei engem und rigidem Scheidenrohr den Widerstand desselben durch einen den Levator ani durchtrennenden Scheidendammschnitt beseitigt.

Unter diesen Umständen lässt sich die Operation auch in einer Dachkammer unter alleiniger Assistenz der Hebamme und mit derselben Aussicht auf guten Erfolg, wie sie eben jeder unter solchen Verhältnissen ausgeführte Eingriff gewährt, ausführen. Das hat Rühl, der die meisten Fälle ausserhalb der Klinik operirt hat, bewiesen. 
Nan führe doch einmal in all den geburtshülflichen Kliniken, in denen die Bossi'sche Methode angewendet wird, den vaginalen Kaiserschnitt aus und man wird dann, dessen bin ich sicher, nie wieder auf die Bossi'sche Methode zurückgreifen.

Es sei mir gestattet, zunächst einen weiteren, von mir ausgeführten vaginalen Kaiserschnitt mitzutheilen, welcher beweist, dass der vaginale Kaiserschnitt trotz Ausziehung des unteren Uterinsegments - gerade durch die ihm eigenthümliche Technik, welche die Spannung des unteren Uterinsegments aufhebt - eine gefahrlose Wendung ermöglicht und für ihn daher bei engem Becken Schwierigkeiton, welehe sich in mehreren der Ehrlich'schen Fälle der Entbindung nach Bossi entgegenstellten, voliständig wegfallen.

J.-No. 347. 14. 10. 04. Frau H., eine 28 jährige I para, hatte die letzte Menstruation am 20. December 1903 und wurde von Herrn Dr. Pautz zum Zweck der Entbindung in meine Klinik geschickt. Sie kreisste bereits seit 40 Stunden, das Fruchtwasser war bereits vor 18 Stunden abgeflossen, und trotz kräftiger Wehen war der Kopf nicht in das Becken eingetreten.

Status: Kräftig gebante, grosse Brünette mit ängstlichem Gesichtsausdruck, einer Temperatur von 38, einem Puls von 120. Der Leib ist stark ausgedehnt, der Contractionsring steht in Nabelhöhe, der grosse Kopf in erster Schädeilage fest auf dem Beckeneingang. Die kindlichen Herztöne betragen 150, es geht Meconium ab.

Beckenmaasse: $29,31,19,10^{1} / 2$.

Die innere Untersuchung ergiebt ein völlig erhaltenes, für 2 Finger durchgängiges Collum, den harten Schädel in Vorderseheitelbeinstellung über dem Beckeneingang. Das Collum war schlaff, wie bei einer Mehrgebärenden, und wohl ursprünglich schon durch die Fruchtblase in seiner supravaginalen Partie entfaltet gewesen, aber nach dem Blasensprung wieder zusammengefallen.

Es handelte sich somit um ein durch das platte Becken and das grosse Kind (Uebertragung) bedingtes Geburtshinderniss, welches zu einer Lebensgefahr der Mutter (Ausziehung des unteren Uterinsegments) und des Kindes geführt hatte. Eine sofortige Entbindung war im Interesse Beider geboten. Die Metreuryse erschien bedenklich wegen der schon vorhandenen Dehnung des unteren Uterinsegments, welch' letztere auch die nachfolgende Wendung zu einem riskanten Lnternehmen gemacht hätte, während andererseits die Zange wegen des Hochstandes des Kopfes nicht möglich war.

Früher hätte ich in diesem Fall - unter Berücksichtigung der Wabrscheinlichkeit, dass die supravaginale Cervixpartie schon entfaltet gewesen war - 4 tiefe Cervixincisionen gemacht und die Wendung noch riskirt, jetzt aber erachtete ich den vaginalen Kaisersehnitt für das sicherste Verfahren und erhoffte auch von der Spaltung des unteren Uterinsegments eine Erleichterung und gefahrlose Durchführung der Wendung, indem ja diese Spaltung die zur -Uterusruptur disponirende 
Spannung des unteren Uterinsegments beseitigen musste - eine Hoffnung, die sich im vollsten Maasse erfüllte.

Operation: Rechtsseitige Scheidendammineision wegen des engen und rigiden Vaginalrohres, Unterbindung einiger spritzender $\mathrm{Ge}-$ fässe. Einstellung der Portio mit 2 grossen Doyen'schen Spiegeln, Durchführung der seitlichen Fadenzügel, Spaltung der hinteren Lippe und eines $2 \mathrm{~cm}$ langen Stückes des hinteren Scheidengewölbes, Abschieben lockeren Gewebes von der hinteren Uteruswand mittelst eines in das eröffnete Scheidengewölbe eingeführten Fingers, Spaltung der vorderen Lippe und eines $5 \mathrm{~cm}$ langen Stückes des vorderen Scheidengewölbes - welches nach den Seiten zu durch einige Scheerenschläge etwas von der Harnblase abpräparirt wird - Abschieben lockeren Gewebes von der vorderen Uteruswand in derselben Weise wie an der hinteren, Spaltung beider Uteruswände. Nunmehr dringt die Hand leicht in den Uterus und wendet mit grosser Leichtigkeit. Der Entwickelung des Steisses setzte die etwas zu kurze Scheidendammincision einigen Widerstand entgegen, auch die Entwickelung des schr barten Kopfes gelang erst in Walcher'scher Hängelage durch den Wiegand-MartinWinckel'schen Handgriff.

Das Kind, ein $4250 \mathrm{~g}$ schwerer und $54 \mathrm{~cm}$ langer Knabe, .war asphyktisch, wurde aber rasch wieder belebt.

Wegen Atonie sofortige manuelle Lösung der Placenta und Uterustamponade. Naht der hinteren Uteruswumde mit 4, der vorderen mit 6 Catgut-Kopfnähten, des vorderen Scheidengewölbes durch 4 Stiche einer fortlaufenden Nabt, während das hintere durch eine der Uterusnähte mitgeschlossen wurde. Tamponade des antecervicalen Hohlraums durch einen Jodoformgazestreifen.

Naht der Scheidenwunde in der üblichen Weise. Scheidentamponade mit Watte.

Die Operation hatte bis zur Extraction des Kindes 10 Minuten, die ganze Operation 40 Minuten gedauert. Es wohnten derselben bei: Meine Assistenten, die Herren Dr. Wolff, Albrecht; Brüning, mein früherer Assistent Dr. Priebatsch (Berlin), Dr. Simon (Schulitz), Dr. Busalla, 1. Assistent der Hebammenlehranstalt zu Hannover, Dr. Löfqu vist, Assistent von Prof. Engström (Helsingfors).

14. 10. Abends Entfernung sämmtlicher Wattetampons und Streifen.

28. 10. Die Wöchnerin wird nach reactionslosem Verlauf mit p. pr. geheilten Portio- und Scheidenwunden und anteflectirtem Uterus entlassen. Das Kind gedeiht gut bei künstlicher Ernährung.

Hat man derartige Fälle von Anfang an unter Beobachtung, so kann man vielfach durch rechtzeitige kleine Eingriffe grosse überflüssig machen.

In Fällen z. B., wie in dem Fall VI der Ehrlich'schen Arbeit, wo die Nephritis in der Schwangerschaft trotz rationeller Behandlung (Bettruhe, Milchdiät, Bäder, Einpackungen) zunimmt, wo die Oedeme und Kopfschmerzen stärker werden, warte ich nicht den Ausbruch der Eklampsie ab, um dann blutige Operationsmethoden anzuwenden, sondern für diese Fälle wende jch mit 
Vorliebe die Metreuryse an. Als Beispiel führe ich folgenden Fall an:

I. J.-No. 235. Anamnese: Am 3. 7. 04 wurde die 36 jährige I gravida Frau Sch. von Herrn Dr. Hentzelt, demselben Collegen, welcher mir bereits einen Fall von vaginalem Kaiserschnitt wegen Eklampsie zugewandt hatte, behufs Vornahme dieser Operation zugeschickt. Frau S. ist soit 3 Jahren verheirathet, die letzte Menstruation hatte sie am 31.10.03. Vor 3 Wochen bekam sie starke Anschwellungen der Beine. Am 29. 6. constatirte der zugerufene Herr Dr. Hentzelt auch Oedeme im Gesicht und Albumen im Urin. Trotz rationeller Behandlung wurde die Urinmenge geringer, die Oedeme steigerten sich. der Eiweissgehalt nahm zu, und am. 3. Tage traten auch beftige Nierenund Kopfschmerzen auf, so dass Herr Dr. H. die Schwangere wegen drohender Eklampsio in meine Klinik schickte.

Aeussere Untersuchung: Mittelgrosse, krätige Frau mit stark gedunsenem Gesicht und geschwollenen Beinen. Der Puls ist kraftig, sebr gespannt, 76. Fundus steht handbreit über dem Nabel, der Kopf in erster Schädellage beweglich über dem Becken. Herztöne normal.

Innere Untersuchnng: Cervix gescblossen und völlig erhalten.

Urin erstarrt beim Kochen und enthält Epithel- and grranulirte Cylinder.

3. 7. Am Nachmittag, einige Stunden nach der Aufnahme, wird die Cervix mit Hegar'schen Dilatatoren bis zu Fingerweite dilatirt, ein Braun'scher Kolpeurynter in den Uterus eingeführt und mit $300 \mathrm{ccm}$ Lysoformlösung gefüllt.

4. 7. Horgens. Es sind kräftige Wehen dagewesen. Der Kolpeurynter wird durch leichten Zug entfernt. Im Crin 1,2 pCt. Albumen.

i. 7. Da die Wehen soit der Entfermung des Kolpenrynters anfgehört haben, wird ein Müller'scher Ballon in den Uterus geführt und mit $1 / 2$ Liter gefüllt. Alsbald stellten sich kräftige Wehen ein, welche das $W$ asser aus dem nicht exact versehlossenen Ballon allmälig austrieben. 5 Stunden später wurde der leere Ballon herausgezogen. Die Wehen dauern fort und beförderten nach weiteren $2^{3 / 4}$ Stunden ein lebendes, ausgetragenes Mädchen zur Welt. 1/2 Stunde später Expressio placentae.

20.7. Die Wöchnerin wird nach normalem Wochenbett ohne Eiweiss entlassen. Das Kind gedeiht bei künstlicher Ernabrung.

In anderen Fällen kommt man mit noch geringeren Eingriffen aus:

II. J.-No. 61. Anamnese: Am 28.2.02 wurde die 30 jährige, am Ende der Schwangerschaft befindliche I gravida Frau A. von Herrn Dr. Pautz meiner Klinik überwiesen, da eine vorhandene Schwangerschaftsnephritis trotz rationeller Behandlung im Zmehmen begriffen war. Wir constatirten eine Eiweissausscheidung von 1.1 pCt., am 1. 3. von $1,2 \mathrm{pCt}$. und an diesem T'age eine Urinmenge von nur $800 \mathrm{ccm}$.

Die Untersuchung ergiebt [2. Schädellage, den Kopf über dem Becken, den Cervicalcanal erhalten und für 1 Finger durchgängig.

Therapie: 1. 3. Eihautstich entleert eine mässige Menge ron Fruchtwasser. 
2. 3, In der Nacht geringe Wehen. Eiweissgehalt $1,3 \mathrm{pCt}$.

3. 3. Nachmittags. Es sind kräftige Wehen dagewesen. Der Muttermund ist zweimarkstückgross, die Portio verstrichen, aber verdickt und rigide. Der Kopf steht fest im Becken.

4. 3. Nachmittags. Der Eiweissgehalt beträgt $1,6 \mathrm{pCt}$. Die Wehen haben fast ganz aufgehört. Der Muttermund ist fünfmarsłückgross, sonst von derselben Beschaffenheit.

Wegen der Zunahme der Eiweissmenge bei geringer Urinsecretion $(800 \mathrm{ccm})$ wird die Entbindung beschlossen und zunächst wegen des rigiden Scheidenrohres eine rechtsseitige Scheidendammincision gemacht. Nach Einstellung der Portio mit 2 breiten Spiegelplatten werden 4 tiefe Cervixincisionen und sodann eine schräge Zange angelegt, welche ein lebensfrisehes, ausgetragenes Mädchen zur Welt befördert. Naht der Cervixincisionen mit im Ganzen 9 Catgutknopfrähten, Expressio placentae, Naht der Sebeidendammincision, in der Scheide mit fortlaufendem Catgutfaden, am Damm mit 3 Silkwormnähten.

5. 3. Nachm. Die Urinmenge ist auf das Doppelte gestiegen. Der Eiweissgehalt beträgt nur noch $1 / 2 \mathrm{pl}$.

20. 3. Hutter und Kind werden in bestem Wohlsein, erstere mit tadellos gebeilten Wunden entlassen.

III. J.-No. 100. An amuese: Die 20 jährige I gravida P. bekam am 20.3. $04 \mathrm{im} \mathrm{7.} \mathrm{Nonat} \mathrm{der} \mathrm{Schwangerschaft} \mathrm{eine} \mathrm{starke} \mathrm{Anschwellung}$ der Beine und wurde von dem consultirten Arzt wegen Albuminurie der Klinik überwiesen.

24. 3. Status: Gesicht stark gedunsen, Labien faustdick geschwollen, Beine ebenfalls sehr stark geschwollen. Fundus in Nabelhöhe. Kopf im Becken, vordere Lippe verstrichen, Cervicalcanal aber erhalten und nicht für einen Finger durchgängig. Urin enthält sehr viel Eiweiss, Leukocyten und hyaline Cylinder, seine Menge ist erheblich vermindert.

Da offenbar schon Wehen dagewesen sind, die Rücksicht auf Erhaltung der Schwangerschaft somit wegfällt, wird am 24. 3. Abends ein Laminariastift eingelegt. Ausserdem erhält die Pat. heisse Bäder mit nachfolgenden nassen Einwickelungen.

25. 3. Der Stift ist spontan ausgestossen. 26. 3. Nach vorausgegangenen Wehen ist der Muttermund markstïckgross geworden, die Portio verstrichen. 27. 3. Spontane Geburt eines nach 24 Stunden verstorbenen Mädchens. 2. 4. Die Pat. wird geheilt entlassen.

Als Gegenstück führe ich einen weiteren von mir bei Eklampsie ausgeführten vaginalen Kaiserschnitt an:

J.-No. 418. Anamnese: Am 26. 12. 04 wurde die 34 jährige, VII gravida Frau H. Abends um $71 / 2$ bewusstlos mit einem Krankenwagen in die Klinik eingeliefert, nachdem sie von Herrn Dr. Pautz schon vor einigen. Monaten wegen Schwangerschaftsnephritis behandelt war und $1 \frac{1}{2}$ Stunden zuvor einen schweren eklamptischen Anfall durchgemacht hatte, nach welchem eine Morphiuminjection verabreicht wurde. Wie nachträglich vom $\mathrm{Mann}$ berichtet wurde, war Pat. bereits $11 / 2$ Stunden vor dem Anfall bewusstlos, nachdem sie vorher wegen Erbrechens das Bett aufgesucht hatte.

Status: Pat. ist eine elende Proletarierfrau, die sich hin und her wirft. Der Puls ist kräftig, die Athmung frei, der Urin enthält einen mässigen Eiweissniederschlag. Fundus in Nabelböhe, Schädellage. 
Keine Wehen. Schwangerschaft im 7. Monat. Portio hypertrophiseh, lacerirt, indurirt. Der Cervicalcanal lässt den Finger nur $1 \mathrm{~cm}$ weit eindringen, seine $W$ and ist ungemein rigide und dick.

Therapie. Chloroformnarkose, Ergotininjection. Vaginaler Kaiserschnitt mit nur einem Assistenten bei der Operation. Die Portio wird mit 2 Doyen'schen Spiegeln eingestellt und mit 2 seitlichen Fadenzügeln versehen. Spaltung der hinteren Lippe und des hinteren Scheidengewölbes. Da das stumpfe Abschieben des Douglas. peritoneums nicht gelingt und ein unteres. Uterinsegment völlig fehlt, wird zur Erweiterung des stenotischen inneren Mlttermundes die Spaltung der hinteren Uteruswand mehrere Centimeter über den inneren Muttermund hinaus fortgesetzt und hierbei der Douglas geöffnet. Spaltung der vorderen Lippe, des vorderen

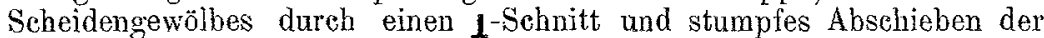
Harnblase mittelst Doyen'schen Spiegels, worauf auch die vordere Uteruswand weit über den inneren Muttermund hinaus gespalten wurde. Die Blutung aus den stark verdickten Uteruswänden war nur gering. Es folgte die Wendung mit der halben Hand und leichte Extraction eines $40 \mathrm{~cm}$ langen Knabens mit 28,5 cm Kopfumfang; welcher bald nach der Geburt starb.

Da der Uterus sich gut contrahirte, so wurden zunächst die Uteruswunden durch Zug an den Fadenzügeln sichtbar gemacht und ihre obere Hälfte durch Catgutnähte versorgt. Hierbei zeigte es sich, dass auch der vordere Douglas eröffnet war. Es folgte 10 Minuten später die leichte Expression der Placenta, an die sich die weitere Anlegung von Nähten und Knüpiung sämmtlicher 12 Nähte anschloss. Schluss des hinteren Scheidenschnitts durch, das Douglasperitoneum mitfassende, querdurchgelegte Catgutknopfnähte bis auf eine hintere Oeffnung, durch welche ein Jodoformgazestreifen in den Douglas geführt wurde. Ein zweiter solcher Streifen wurde durch die nicht vernähte vordere Scheidenwunde in den vorderen Douglas geführt. Scheidentamponade mit Watte. Dauer der ganzen Operation $1 / 2$ Stunde.

Verlauf. Abends um $10^{1 / 2}$ zeigt Pat. schon auf Aufforderung die Zunge.

27. 12. Morgens. Pat. hat wegen Unruhe 2 Morphiuminjectionen a 0,02 erhalten. Sie ist bei Bewusstsein und klagt nur über Durst. Blutung war überhaupt nicht vorhanden. Urin hat die Wöchnerin noch nicht gelassen. Der Leib ist weich, der Fundus steht in Nabelhöhe. Temp. 36,4; Puls 72, kräftig. Entfernung der Watte und der Drainagestreifen.

28. 12. Wohibefinden. Pat. giebt an, dass sie sich seit dem Abend des 24. auf nichts besinnen kam, obgleich die Eklampsie erst 2 Jage später ausbrach.

4. 1. 05. Absolut reactionslaser Verlauf. Urin eiweissfrei.

8. 1. 05. Keine Beschwerden, nachdem die Wöchnerin am 7. aufgestanden ist.

Status: Uterus gut zurückgebildet, anteflectirt. Portio- und Scheidenwunden lineär vernarbt, kaum sichtbar. - Geheilt entlassen (am 12. Tag).

Den zweiten Fall von vaginalem Kaiserschnitt bei Eklampsie habe ich mitgetheilt, um an ihm die Ueberlegenheit des 
vaginalen Kaiserschnitts über alle anderen Dilatationsmethoden zu demonstriren. Es handelte sich um einen schweren Fall von Eklampsie bei wirklicher SchwangerschaftsNephritis. Dem ersten Anfall war bereits eine Bewusstlosigkeit von $1 \frac{1}{2}$ stündiger Dauer vorausgegangen, und der Anfall selbst war nach der Mittheilung des behandelnden Arztes, der ihn beobachtete, ein schwerer. Es war also Eile geboten. LaminariaDilatation des rigiden und engen Collum kam somit nicht in Betracht, die Einführung irgend eines Gummiballons oder eines Bossi-Instruments war nicht möglich. Hätte man letzteres nach vorausgeschickter Dilatation mit Hegar'schen Dilatatoren vielleicht einführen können, so hätte es mit absoluter Sicherheit grosse Zerreissungen gemacht, da das Collum hypertrophisch und ungemein rigid war.

Der vaginale Kaiserschnitt ging dagegen trotz weniger Assistenz glatt und leicht von Statten, und trotz Eröffnung der Bauchhöhle verlief die Reconvalescenz wie ein normales Wochenbett, so dass Pat. bereits am 12. Tag mit kaum noch sichtbaren, lineären Narben und anteflectirtem Uterus entlassen werden konnte.

Die von Ehrlich veröffentlichten 30 Fälle aus der Leopoldschen Klinik bringen ebenso wenig wie die früheren 17 Fälle den Beweis für die Ueberlegenheit ja nur für die Gleichwerthigkeit der Bossi'schen Methode gegenüber anderen Methoden. In den Fällen I, III, XXVI, XXVII, XXX handelte es sich um Erstgebärende mit verstrichener Portio und in das Becken eingetretenem Kopf, der Muttermund war zehnpfennig- bis markstückgross. In diesen Fällen hätten die tiefen Cervixineisionen des Verfassers in kürzerer Zeit die Entbindung ermöglicht (ef. Fall II des Verfassers). In den Fällen XXI, XXIV, XXV hätte die Perforation, da der Muttermund zwei- bis fünfmarkstückgross war, auch ohne vorherigen Bossi gemacht werden können. In den Fällen VII, VIII, XI, XII, XIX, in welchen keine Eklampsie bestand und keine solche Eile nöthig war, hätte die sofort eirigeleitete Metreuryse mit dem Braun'schen Kolpeurynter oder dem Müller'schen Ballon mit constantem selbstthätigen Zug mindestens dieselbe Dilatation, wie die Bossi'sche Methode, aber schonender erzeugt. Im Fall XVII konnte die Metreuryse mindestens so lange versucht werden, bis ein eklamptischer Anfall auftrat. Mit dem Ballon hätte man alsdann wohl ebenso rasch und ebenso weit die Cervix dilatiren 
können, als mit dem Bossi'schen Instrument, welches bis $71 / 4$ erweitert.

In folgenden Fällen zeigte sich direct die Unvollkommenheit der Bossi'schen Methode, indem sie nicht anwendbar war oder keine völlige Dilatation zu Wege brachte und hierdurch der Mutter oder dem Kind directe Schäden zufügte:

In Fall II war die hohe Zange schwer wegen ungenügender Dilatation, welche eine einstündige Narkose erforderte. Eine Operation, welche nur eine Narkose von 5 Minuten Dauer erfordert, wie die Sectio caesarea vaginalis, hätte die schwerkranke Eklamptische bestimmt weniger geschädigt, wenn ich auch nicht sagen kann, dass sie jhr das Leben gerettet baben würde. Bei der Section fand sich ein tiefer Collumriss.

In Fall IV erzengte der nachfolgende Kopf wegen der mangelnden Dilatation einen Collumriss, der mit einer Umstechung geschlossen wurde.

In Fall V, bei dem rigiden und geschlossenen Collum einer 34 jährigen I para, hätte der vaginale Kaiserschnitt 3 Tage früher als die Bossi'sche Methode ausgeführt werden können und mit Sicherheit ein lebendes Kind ergeben.

In Fall VI konnte die Dilatation des fingergliedlangen Scheidentheils wegen eintretender Blutung nicht viel über 6 getrieben werden. In Folge dessep konnte die Eklamptische nicht, wie beabsichtigt, entbunden werden, und das combinirt gewendete Kind starb ab. Es entstanden zwei tiefe Collumrisse, die durch je 6 Nähte vereinigt wurden. Der vaginale Kaiserschnitt hätte hier mit Sicherheit ein lebendes Kind erzielt.

In Fall $X$ Ironnte ebenfalls wegen wulstiger und rigider Portio der Muttermund nicht völlig dilatirt werden. Die Wendung gelang daher nieht, und das Kind wurde perforirt. Die Mutter trug einen Collumriss davon, der mit 2 Nähten vereinigt wurde.

In Fall XIII konnte der nur für eine Fingerkuppe zugängige Muttermund der fast verstrichenen Portio ebenfalls nur bis Handtellergrösse erweitert werden. Die Entbindung mit hoher Zange war daher nicht möglich, und das Kind wurde perforirt. Die Mutter trug einen Collumriss davon, der mit 2 Nähten vereinigt wurde.

In Fall XIV konnte die Eklamptische des 7. Monats erst 3 Stunden nach der Einlieferung entbunden werden, da das Frommer'sche Instrument sich nicht durch den inneren 
Muttermund bringen liess. Man musste daher die Erweiterung zunächst den Wehen überlassen und konnte erst zwei Stunden nach der Einlieferung mit dem Bossi'schen Instrument erweitern. In dieser Zeit hatte die Pat. die Schädigung von sechs weiteren Anfällen und der protrahirten Morphium-Chloroformnarkose $z u$ überstehen. Dic Pat. starb.

In Fall XV konnte der für einen Finger durchgängige Muttermund wegen eintretender Blutung in Folge tiefen Sitzes der Placenta nur bis $7 \mathrm{~cm}$ erweitert und die Eklamptische konnte daher trotzihres bedrohlichen Zustands nicht entbunden werden. Erst 7 Stunden später wurde die Zange angelegt. Das Kind starb -- jedenfalls in Folge dieser Verzögerung der Entbindung.

In Fall XVI konnte die Dilatation des bereits markstückgrossen Muttermundes bei wulstiger Portio nur bis $7 \frac{1}{2}$ getrieben werden. Natürlich misslang bei dem nur handtellergrossen Muttermund die hohe Zange, und das lebende Kind wurde perforirt.

In Fall XVIII, bei einer Eklamptischen des 7. Monats, erforderte die Dilatation, die zunächst mit dem Bossi'schen Instrument nicht möglich war, und die schwierige Extraction des perforirten Kindes 2 Stunden. Der vaginale Kaiserschnitt hätte nur 5 Minuten erfordert, 15 Stunden früher ausgeführt werden können und hierdurch der Kranken, welche starb, manche Schädigungen erspart.

In Fall XX wurde die halbfingergliedlange Portio bis auf $91 / 2$ dilatirt, der Muttermund umschloss aber nach der Wendung den Hals des Kindes so fest, dass es perforirt werden musste. Ein Collumriss erforderte eine Naht.

In Fall XXII, in welchem Mutter und Kind starben, wäre das Kind durch vaginalen Kaiserschnitt lebend zur Welt gebracht worden.

In Fall XXIII konnte der schon zweimarkstückgrosse Muttermund einer II para nur bis $81 / 2$ erweitert werden, da ein später durch 3 Nähte geschlossener Collumriss eine Blutung erzeugte.

In Fall XXVIII kam das Kind tief aspbyktisch zur Welt, weil die Zange in Folge des Widerstandes des Muttermundes schwierig war.

In Fall XXIX erzeugte das bei handtellergrossem Muttermund einer II para angelegte Frommer'sehe Instrument 2 Collumrisse, die 5 Nähte erforderten. Das Kind hätte nach vaginalem Kaiserschnitt wahrscheinlich nicht perforirt zu werden 
brauchen. Jedenfalls dürfte sich in einem solchen Fall - auch der Fall XXIV fällt in diese Kategorie - nach dem Resultat des von mir in dieser Arbeit mitgetheilten vaginalen Kaiserschnitts der vaginale Kaiserschnitt als rationeller erweisen, da er nicht nur das Collum vollständig dilatirt, sondern auch die Dehnung des unteren Uterinsegments beseitigt. Hierdurch fallen alle Hindernisse bezw. Contraindicationen der Wendung bei engem Becken fort, und es wird sich durch die angeschlossene Extraction marches Kind lebend entwickeln lassen - zumal bei mässig verengten Becken, wie in Fall XXIV und XXIX, in denen schon eine spontane Geburt vorausgegangen war.

Wie soll sich, frage ich, der praktische Arzt in solchen Fällen, wie Fall XIV und XVIII verhalten, in welchen die Einführung des Bossi'schen oder eines anderen Dilatators wegen der Enge des Cervikalcanals. nicht gelingt? Es ist doch nicht anzunehmen, dass ein Arzt, der wirklich das Bossi'sehe Instrument seiner geburtshilflichen Tasche einverleibt hat, daneben noch andere Dilatatoren mit sich führt? Wozu auch? Bossi hat ibn doch versichert, dass sein Instrument in allen, auch den verzweifeltsten Fällen den Arzt zum Herrn der Situation mache!

Nun für den practischen Arzt ist es ein Glück, wenn ihm in solchen Fällen die Einführung des Bossi'schen Instrumentes überhaupt nicht gelingt. Denn in diesen Fällen von erhaltenem Collum, die gerade den Prüfstein für den Werth einer Dilatationsmethode abgeben, versagt, wie die Leopold'schen und andere Fälle beweisen, sogar in der Klinik, dio Methode meistens, die Dilatation ist eine sehr zeitraubende, gelingt trotzdem nur unvollkommen, und die weitere Folge dieser mangelhaften Dilatation ist die Entstehung von Cervixrissen bei der Extraction bezw. das Misslingen der Extraction eines lebenden bezw. unzerstïekelten Kindes.

Ich wende mich nunmehr zu einer kritischen Betrachtung der von Ehrlich aufgestellten. Indicationen zur Dilatation und Entbindung nach Bossi.

Wenn Ehrlich der Bossi'schen Methode bei Eklampsie den Charakter einer specifischen Therapie beimisst, weil die Methode sich hier auf das Glänzendste bewährt hat, so muss ich dieser Behauptung auf das Entschiedenste widersprechen. Die sperifisehe Therapie besteht in der zuerst von dem Verf. principiell geforderten sofortigen Entleerung des Uterus nach dem ersten beobachteten 
eklamptischen Anfall. Würde die Bossi'sche Methode diese sofortige Entleerung in allen Fällen und ohne Gefahr für die Mutter und das Kind zu Wege bringen, dann könnte man die Bossi'sche Methode als eine specifische Therapie der Eklampsie unter der Voraussetzung bezeichnen, dass keine anderen Methoden vorhanden wären, die dasselbe leisteten. In der That behauptet ja Ehrlich:

"Gerade das unbedingte Gelingen der durch die ernste Erkrankung gebotenen Entbindung ohne erheblichere Gefährdung des mütterlichen Lebens, die ausserordentliche, für die eklamptische Vergiftung so bedeutsame Schnelligkeit der Entleerung des Uterus, die Möglichkeit der Anwendung des Dilatators zu jeder Zeit der Schwangerschaft, sowie seine relativ einfache Handhabung, sind geeignet, der Bossi'schen Methode einen hervorragenden Werth und bedeutsamen Vorzug vor den übrigen Verfahren in der Behandlung dieser schweren Krankheit zu verleihen."

$\mathrm{Zu}$ diesen Behauptungen ist Ehrlich auf Grund der von ihm mitgetheilten 30 Fälle ganz und gar nicht berechtigt: Die Entbindung mittels der Bossi'schen Methode gelingt nicht unbedingt. Denn in den Fällen VI, XIV, XV gelang sie nicht, vielmehr musste die Dilatation unterbrochen und $\mathrm{zu}-$ nächst die weitere Geburt den Wehen überlassen werden. Sämmtliche Kinder und eine Mutter starben. In weiteren Fällen gelang sie ebenfalls nicht in idealer Weise, führte vielmehr in Folge der mangelhaften Dilatation (in Fall IV, X, XIII, XX, XXIII, XXIX) zu Collumrissen, also zu einer Gefährdung des mütterlichen Lebens, bezw. zum Tode des lebensfähigen Kindes, weil Zange oder Wendung in Folge der mangelhalten Dilatation versagten und daher für die Entleerung des Uterus nur noch die Perforation in Betracht kam (in Fall X, XIII, XVI, XX, XXIX).

Auch klingt es wunderbar, von der ausserordentlichen Schnelligkeit der Entleerung des Uterus zu sprechen - bei einer Methode, welche unter Umständen überhaupt nicht gelingt, in anderen Fällen eine Dauer bis zu drei Stunden in Anspruch nimmt, während Methoden existiren, die, wie der vaginale Kaiserschnitt, mit unbedingter Sicherheit den Uterus in fünf Minuten entleeren können. Es giebt dahier auch bei Fieber der Mutter sicherere und schneller wirkende Methoden als die Bossi'sche.

Warum liess man es überhaupt in Fall $V$ zur Entstehung des Fiebers kommen? Wenn man Gefahr für das Kind als Indication 
für die Anwendung der Bossi'schen Methode ansieht, dann hätte man in diesem Fall die Methode drei Tage früher anwenden sollen. Allein man befürchtete wohl, dass die Methode bei dem rigiden und geschlossenen Collum der 34 jährigen I para nicht gelingen würde. Der vaginale Kaiserschnitt hätte hier mit Sicherheit ein lebendes Kind erzielt. In den beiden anderen Fällen ron Fieber konnte man die Entleerung des Uterus durch die Perforation bezw. die Zange schneller und schonender mit Hülfe ron zwei tiefen Cervixincisionen bewirken. Denn Einrisse, die durch vier bezw. drei Nähte veremigt wurden, gab es ja doeh in beiden Fallen (XXV und XXVII) bei der Bossi'schen Vethode.

Dass die Bossi'sche Methode bei Stillstand der Goburt in Folge engen Beckens, and zwar wesentlich im Interesse des Kindes, oder bei alleiniger Gefährdung des kindlichen Lebens empfehlenswerth sei, beweisen die Fälle von Ehrlich ebenso wenig. In keinem der Fälle von engem Becken (VIII, XII, XXIV, XXIX) konnte das kindliche Leben erhalten werden, und in den zwei Fällen der anderen Kategorie hätte im Fall XIX der Metreurynter auch ohne vorherige Bossi-Dilatation eingeführt werden können, wäbrend in Fall XXIII das Krull'sche Dilatatorium einen Cervixriss erzengte und die hohe Zange daher bei mangelhaft erweitertem Muttermund angelegt werden musste.

Bei vorzeitiger Lösung der normalsitzenden Placenta oder als Ersatz des Kaiserschnittes in agone et in mortua kann nach Ansicht des Verf.'s die Bossi'sche Methode wegen ibrer Unsicherheit und langsamen Wirkung ebensowenig empfohlen werden, wenn man bedenkt, dass der vaginale Kaisersehnitt in diesen Fällen binnen weniger Minuten die schonende Entleerung des Uterus ermöglicht. -

In dem Kapitel, Zeitpunkt der Schwangerschaft, Erstund Mehrgebärende, sagt Ehrlich: "Uns ist zu jedem Zeitpunkt der Schwangerschait, sobald dieser nur sich für das Verfahren geeignet bewies (vom 6. Monat ab), auch bei Erstgebärenden unter poinlichster Berïeksichtigung der genannten jeweiligen anatomischen und physiologischen Verhältnisse die instrumentelle Exweiterung des Muttermundes gelungen; wir können daher die oben angefühte Behauptung Bossi's vollauf bestätigen."

Zwei Fälle von Ehrlich widersprechen dieser Behauptung. Fail XIV war ein eklatanter Misserfolg (s. 0.), und in Fall XVIII war selbst die Extraction einer Frucht aus dem 7. Monat erst 
nach zweistündiger Operation und Zerstückelung des Kindes unter Schwierigkeiten möglich. -

In dem Kapitel, Verhalten des Muttermundes bezw. der Cervix vor, während und nach der Dilatation, werden die Schwierigkeiten und Gefahren der Bossi'schen Iethode bei erhaltenem Collum zugegeben, so dass sie in solchen Fällen nur aus den seltensten und dringlichsten Anlässen angewandt werden soll. Es wird auch schon angedeutet, dass in solchen Fällen noch ein anderes Dilatationsverfahren, welches Verf. für sehr wenig empfehlenswerth hält, nämlich die hohe Zange, angewandt werden muss, um das Zusaminenschnappen des durch einen Bossi-Dilatator erweiterten Muttermundes zu verhüten. Während also für diese Fälle, bei denen der Kopf naturgemäss noch über dem Becken steht, die Wendung die gegebene Operation ist, wird die für solche Fälle garnicht passende Zange angewandt, weil man durch die Zangenextraction den Muttermund manchmal mittels forcirten Zugs erweitern kann, während die der Wendung nachfolgende Extraction dazu führen kann, dass der mangelhaft erweiterte Muttermund um den Hals des Kindes zusammenschnappt, und dass das Kind abstirbt.

Wenn Ehrlich in der Technik der instrumentellen Dilatation sagt: Man solle nach bewirkter Dilatation sich den Muttermundssaum ansehen, $n 1 \mathrm{~m}$ etwa, nach der Entwickelung des Kindes aufgedeckte Verletzungen der Cervix nicht ungerechtfertigter Weise dem Dilatator zuzuschreiben", so ist demgegenüber zu sagen, dass solche Risse, wenn sie auch erst durch die Entwickelung des Kindes entstehen, selbstverständlich das Conto der Bossi'schen Methode belasten. Sie sind eben ein Beweis für die Unzulänglichkeit der Dilatationsmethode, welche keine völlige Dilatation der Cervix zu Wege brachte.

In dem Kapitel, Operationen im Anschluss an die Dilatation, Prognose für Mutter und Kind, sind die statistischen Angaben anders zu ordnen, falls man sich ein Bild über den Werth der Bossi'schen Methode machen will. Ich finde zunächst nicht 19, sondern 21 Zangenextractionen mit 6 gestorbenen Kindern, die vor der Dilatation noch lebten. 3 dieser Kinder (Fall II, IV, XI der ersten Leopold'schen Arbeit) waren allerdings unreif, 3 andere jedoch reif (Fall XV, XXII, XXVII der Ehrlich'schen Arbeit) und von diesen starb sicher ein Kind (XV) in Folge des Versagens der Bossi'schen Methode. Dieser Methode fallen ferner die 3 Kinder zur Last, bei denen zunächst ein 
Zangenversuch gemacht warde (Fall IX von Leopold, Fälle XIII und XVI von Ehrlich), ferner die Fälle VI, VII, VIII, XX von Ehrlich, in denen der Muttermund vor dem nachfolgenden Kopf zusammenschnappte, möglicherweise auch die Fälle XXIV und XXIX von Ehrlich, in denen (s. o.) der vaginale Kaiserschnitt jedenfalls die Wendung ermöglicht hätte.

Von 25 lebensfähigen Kindern, die yor der Dilatation noch lebensfrisch waren, starben 10 , oder sagen wir won 241.) (unter Ausschaltung des Falles XXIV) 9 in Folge der Bossi'schen Methode, welche durch andere Methoden, speciell den vaginalen Kaiserschnitt, hätten gerettet werden können. Es starben also 37,5 pCt. der Kinder nur in Folge der Bossi'sehen Methode.

Von den 31 eklamptischen Müttern starben 7 (22,6 pCt.). Diese Zahl ist also nicht wesentlich günstiger als die, welche ich ${ }^{2}$ ) aus einer älteren Statistik der Charité (von 1880-1892) gewonnen habe, nach welcher unter 80 Fällen von operativer Entleerung des Uterus 23,75 pCt. starben.

Auch die ron Pollak ${ }^{8}$ ) veröffentlichte Statistik über 71 Bossifälle bei Eklampsie ist nicht wesentlich günstiger. Ziehen wir die ersten 12 Falle ron Leopold ab, welche in dieser Statistik enthalten sind, so bleiben 59 Fälle mit 12 Todesfällen (20 pCt.).

Wo bleibt da der günstige Effect der Bossi'schen Methode? Denn was Schwere der Erkrankung anbelangt, können die hiesigen Fälle denen der Dresdener Klinik wohl gleichgestellt werden. Ferner möchte ich hervorheben, dass ich jetzt 137 Fälle von vaginalem Kaiserschnitt zusammengestellt babe, von welchen 19 Fälle (also noch nicht 14 pCt.) starben. Dabei erzielt der vaginale Kaiserschnitt mit absoluter Sicherheit ein lebendes Kind, falls er nach meinen Vorschriften ausgeführt wird.

Unter diesen 137 Fällen befanden sich 52 Fälle ron Eklampsie mit 12 Todesfällen $(23 p \mathrm{Ct}$.). Von diesen Fällen kann man mit Sicherheit sagen, dass sie durchschnittlich schwerere waren, als die Fälle der Dresdener Klinik, weil es sich durchweg um Fälle mit

1) Es sind dies die Kinder der Fälle 5, 6, 9, 10 der ersten Leopoldschen Arbeit, der Fälle 1, 5, 4, 5 der zweiten Leopold'sehen Arbeit, der Fälle 1, 2, 3, 6, 7, 8, 9, 12, 13, 15, 16, 20, 23, 28, 29, 30 der Ehrlich'schen Arbeit.

2) Dieses Archiv. Bd. 43 .

3) M. f. G. Bd.XX. H. 3 . 
erhaltenem Collum, also um Fälle von Eklampsie in der Schwangerschaft oder zu Beginn der Geburt handelte - Fälle, die erfahrungsgemäss die schlechteste Prognose geben. Zudem wurde in diesen Fällen ${ }^{1)}$ die Prognose zum Theil dadurch getrübt, dass die meisten Operateure zum vaginalen Kaiserschnitt erst sehr spät, als ultimum refugium, griffen - im Gegensatz zu den Fällen der Dresdener Klinik, wo im Vertrauen auf die Bossi'sche Methode die Entleerung des Uterus früher angestrebt wurde. So bin ich überzeugt, dass die Dresdener Klinik, falls sie das richtige Princip der EklampsieBehandlung, nämlich die frühzeitige Entleerung des Uterus, weiterhin - aber unter Anwendung des vaginalen Kaiserschnitts - cultiviren wird, ihre Resultate, sowohl für die Mütter als auch die Kinder, viel bessere sein werden, als jetzt bei der Bossi'schen Methode. Für die Richtigkeit dieser Anschauung sprechen bereits die Erfolge von Bumm.

Uebrigens giebt es auch Anhänger der Bossi'schen Methode, die in besonders schwierigen Fällen den vaginalen Kaiserschnitt vorziehen. So verdanke ich Herrn Professor Leopold Meyer (Kopenhagen) folgende briefliche Mittheilung: „Sie dürfen nicht glauben, dass ich so einseitig bin, dass jch die von Ihnen angegebene Methode nie versucht habe. In 2 Fällen von Eklampsie, die mir für "Bossi" ungeeignet erschienen, haben wir den vaginalen Kaiserschnitt (mit Erfolg) gemacht."

Wenn Ehrlich meint, dass ich mich mit meiner abfälligen Kritik der Bossi'schen Methode in Widerspruch zu sämmtlichen anderen Autoren setzte, so ist das ein Irrthum, welcher nur durch eine grosse Voreingenommenheit für die Bossi'sche Methode erklärt werden kann. Denn selbst Schürmann, dessen Ausführungen nach Ehrlich meine Vorwürfe entkrälten sollen, ist ein schlechter Kronzeuge für ihn, da er die Bossi'sche Methode bei erhaltenem Collum am Ende der Schwangerschaft verwirft und bei Fehlgeburten nur empfiehlt, wenn das Collum aufgelockert und für einen Finger durchgängig ist. In letzterem Fall kann man aber auch mit einem Finger den Uterus ohne Zuhülfenahme der Bossi'schen Methode ausräumen oder bei Blutungen ruhig zuwarten, nachdem man den Uterus tamponirt und das Collum mit Laminariastiften dilatirt hat.

1) Diese Fälle werden von mir demnächst in dem von Winckel'schen Handbuch der Geburtshülfe (Capitel „vaginaler Kaiserschnitt") veröffentlicht werden. 
Nach 12-24 Stunden ist der Uterus dann bequem für 2 Finger durchgängig geworden, so dass man die Frucht unzerstückelt bequem extrahiren kann, falls sie nebst der Nachgeburt inzwischen nicht schon spontan in die Scheide ausgestossen ist. Denn die dynamische Wirkung dioses combinirten Verfahrens ist eine viel energischere als die des Bossi'schen Instruments.

Ich kann also aus dem Schürmann'schen Artikel nur den Schluss zichen, dass dieser Autor für die wirklich schwierigen Fälle, diejenigen, in welchen bei geschlossenem und erhaltenem Collum die Entbindung schnellstens vorgenommen werden muss - also für diejenigen Fälle, in welchen der vaginale Kaiserschnitt nie versagt - die Bossi'sche Methode verwirt. Wern Ehrlich ferner mit Sehürmann hervorhebt, dass in dem einen Ehlampsiefall von Schürmann die Bossi'sche Methode dem Kind das Leben gerettet hat, weil Schürmann sonst perforirt hätte, so ist dem entgegenzuhalten, dass bei in der Beckenweite stehendem Kopf, bei verstrichener Portio und markstïckgrossem Muttermund heutzatage die Berechtigung der Perforation eines lebenden Kindes onter keinen Umständen aberkannt werden kann, selbst wenn die Portio dicksaumig ist. Gerade bei einer dicksaumigen Portio einer alten I para. ist die Bluturg nach Anwendung meiner 4 ticfen Cervixincisionen ganz ausserordentich gering (of. meinen Fall I). Wer aber, wie Sohürmann, hierbei vor schweren Blutungen Sorge hat, der indicire doch, wie $Z$ weifel es empfiehlt, zwischen 2 Klemmen, die ein paar Minuten Jjegen bleiben. Ob man einen solehen Schnitt nur $5-6$ oder nur $1 \mathrm{~cm}$ lang macht; ist doch für die Infectionsgefahr gleichgültig, und bezüglich der Gefahr des Weiterreissens der Schnitte hebt es ja: Sebürmann selbst hervor, dass diese besonders gross ist, falls die Schnitte entgegen meiner Vorschrift zu klein angelegt werden.

Wollte ich mir übrigens die sonderbare Argumentation mancher Bossi-Schwälmer zu eigen machen, so könnte ich sagen; Meine Cervixincisionen erweitern bei verstrichener Portio den Muttermund vollständig, obne dass hierbei tiefe, d. h. in den supravaginalen Collumabschnitt reichende Läsionen entstehen; werden diese nach der Entleerung des Uterus gefunden, so sind sie nicht auf das Conto der Dilatationsmethode, sondern der nachfolgenden Extraction zu setzen! So schliesse ich allerdings nicht, sondern ich verlange von einer brauchbaren Dilatationsmethode, dass sie die leichte Ex- 
traction eines lebenden und lebensfrischen Kindes ermöglicht, und dass andererseits die Extraction bei der blutigen Dilatation keine anderen Verletzungen erzeugt, als sie schon vorher angelegt waren - oder (bei der mechanischen Dilatation) keine Verletzungen erzeugt, die in den supravaginalen Collumabschnitt hinaufreichen. Es stimmt auch nicht, wenn Ehrlich Wirtz gegenüber betont, die Zangenextraction sei in der Dresdener Klinik nach der Bossi'schen Methode stets mögljch gewesen: Denn thatsächlich ist doch in der Dresdener Klinik in 2 Fällen (XIII und XVI) die Zange nicht gelungen, sondern das Kind, welches natürlich durch die vorausgegangenen Zangenversuche bei mangelhaft erweitertem Collum schwer gelitten hatte und hierdurch in den Zustand des Absterbens gelangt war, perforirt worden. Der Tod des Kindes fällt hier gleichfalls der unvollkommenen Dilatationsmethode zur Last!

Dass die Beurtheilung der Bossi'schen Methode Seitens vieler Autoren eine andere ist, als Ehrlich es voraussetzt, beweist auch der Jahresbericht von Frommel über das Jahr 1903. In demselben sagen Sellheim und K. Hegar in dem Capitel "Eklampsie" Folgendes: "Im Allgemeinen gewinnt man den Eindruck, als mache sich auf die erste Begeisterung für den Bossi bereits ein Rückschlag geltend. Cervixrisse werden immer häufiger beobachtet." Weiter äussert sich von Franqué folgendermaassen: "Die grosse Verletzungsgefahr wird in zahlreichen Mittheilungen bestätigt und mit Fällen belegt. Sie scheint auch bei der allerseits und insbesondere von Bossi selbst geforderten langsamen Dilatation unter stetiger Controle mit dem Finger zu bestehen. " "Mit Recht wird verschiedentlich betont, dass die Dilatation zu den grossen geburtshülflichen Eingriffen za rechnen sei, die nur von geübten Geburtshclfern mit genügender Assistenz versucht werden dürfe." Auch Stumpf sagt in dem Capitel ngerichtsärztliche Geburtshülfe": "Die Verletzungen, welche durch die Dilatation des Muttermundes und der Cervix durch das Bossi'sche Instrument hervorgerufen werden, werden von allen Autoren, welche sich über die Verwendung der genannten Erweiterungsmethode äussern, besprochen. Die Literatur über die Bossi'sche Dilatation weist bereits zahlreiche Fälle von tieferen Verletzungen auf."

Alle die Bedenten, welche ich ohne eigene Anwendung der Bossi'schen Methode gegen dieselben ins Feld geführt habe, finden 
ihre eingehende Begründung in der Arbeit von v. Bardeleben ${ }^{1}$ ). Dieselbe ist auch deswegen besonders werthvoll, weil dieser Autor sehr genau beobachtet hat und eigene Erfahrungen über alle in Betracht kommenden Dilatationsmethoden besitzt. Auf Grund dieser sagt er Folgendes: "Ist also dies schnelle mechanisch-instrumentelle Dilatationsverfahren bei entfalteter Cervix insofern relativ ungefährlich, als die Verletzungen, die dabei entstehen können, gewöhnlich nur den Muttermundssaum betreffen, so ist es andererseits dabei entweder überflüssig oder unvollkommener als die Ballonmethode mit Handzug, wenn der Muttermund weich und nachgiebig ist, oder aber unvortheilhafter und unzurerlässiger als sachgemässe Muttermundsineisionen, wenn derselbe rigide ist. "

Bei erhaltenem Collum ist das Misslingen der schnellen Dilatation mittelst Ballons nach v. Bardeleben ein Fingerzejg dafür, dass eine künstliche Erweiterung (auch mit dem Bossi'schen Instrument) ohne Verletzungen nicht möglich sein wird. Cnter diesen Umständen aber "werden wir," so fährt v. Bardeleben fort, "in der Wahl des Verfahrens keinen Augenblick in Zweifel sein können. Wir werden den vaginalen Kaisersehnitt nach $D$ ührssen anwenden, lieber einen geraden Schnitt durch die Nitte der vorderen Cervixwand legen, als ein Verfahren forciren, von welchem wir nunmehr wissen, dass aller Wahrscheinlichkeit nach, wenn wir es weiter fortsetzen, schwere Verletzungen entstehen an Orten und von einer Beschaffenheit, die wir nicht porher bestimmen können, die aber möglicher Weise viel gefährlicher und sicherlich für die Therapie unzugänglicher sind. Dem Ausspruch Knapp's: „Bei den Incisionen muss es, bei Bossi's Verfahren kann es bluten" möchten wir den Satz gegenüberstellen: Bei Bossi's Verfahren müssen Verletzungen entstehen, wenn es da angewandt wird, wo Incisionen am Platze sind."

Ich bemerke hierzu noch ergänzend, dass $v$. Bardeleben in einem soeben erschienenen Artikel ${ }^{2}$ ) meine früheren Mittheilungen über die geringfügigen Veränderungen, welche der vaginale Kaiserschnitt setzt, bestätigt. Andererseits darf ich die Thatsache nicht unerwähnt lassen, dass man trotzdem, aus Besorgniss vor späteren Geburtscomplicationen, den vaginalen Kaiserschnitt perhorrescirt:

1) Dieses Archiv. Bd. 70. H. 1.

2) Heilungsresultate nach vorderem Gebärmutter-Scheidenschnitt and deren Bedeutung für: die Bewerthang dieses Schnellentbindungsverfahrens. Centralbl. f. Gyn. 1904. No. 46. 
Aus der Baseler Klinik hat neuerdings Labhardt ${ }^{1}$ ) einen Warnungsruf gegen den vaginalen Kaiserschnitt ergehen lassen auf Grund der Befürchtung, dass die Narben nach vaginalem Kaiserschnitt Veranlassung zu einer Uterusruptur geben könnten. Er sagt in dieser Arbeit, wie auch v. Bardeleben und Hammerschlag ${ }^{2}$ ), dass eine Geburt nach vaginalem Kaiserschnitt nicht beobachtet sei. Dies ist ein Irrthum. Ich habe in der Encyklopädie der Geburtshülfe von Sänger und von Herff bereits im Jahr 1900 eine Geburt nach dieser Operation mitgetheilt (Capitel "Vaginaler Kaisersehnitt", S. 475) und über dieselbe Folgendes gesagt: „Dass dié Incisionen des unteren Uterinsegments keine Gefahr für spätere Geburten bedingen, beweist der erste Fall des Referenten, welcher sich am 27.11. 99 abermals zur Entbindung stellte. Die Cervix war bereits durch Geburtswehen verkürzt, für einen Finger durchgängig und stand bedeutend tiefer, als das rorige Mal, das Kind lag in erster Querlage: Einführung des Kolpeurynters in den Uterus. Derselbe wurde 6 Stunden später ausgestossen, wobei die Blase sprang. Leichte Wendung und Extraction eines lebenden, kräftigen Mädchens. Wochenbett normal. Am 7. 12. 99 wurde Wöchnerin mit anteflectirtem Uterus entlassen."

Auch Jerie (s. Centralbl. für Gyn. 1905. No. 7) berichtet aus der Pawlik'schen Klinik über eine spontane Geburt 2 Jahre nach einem wegen Eklampsie ausgeführten vaginalen Kaiserschnitt.

Diese Fälle beweisen also, dass eine Geburt nach vaginalem Kaiserschnitt ohne die geringste Störung verlaufen kann. Die Gründe von Labhardt, nach denen ein ungünstiger Verlauf in solchen Fällen zu befürchten ist, sind zudem gar nicht beweiskräftig; denn die Narben des klassischen Kaiserschnitts und der Uterusrupturen bieten ganz andere Verhältnisse dar als wie die Narben des vaginalen Kaiserschnitts. Welche Momente führen eine Ruptur von Kaiserschnittsnarben herbei? Es sind folgende:

1. Mangelhafte Naht,

2. Septische Infection,

3. Insertion der Placenta an der Narbe [Fälle von Guillaume, Everke, Meyer, Ekstein $\left.{ }^{3}\right)$ ],

4. Ueberdehnung des Uterus (durch Zwillinge etc.).

1) Ztschr. f. Geburtsh, u. Gyn. Bd. 53. H. 3.

2) Monatsschr. f. Geburtsh. u. Gyn. Bd. 21. H. 1.

3) Centralbl. f. Gyn. 1904. No. 44. 
ad 1. Die mangelhafte Naht erklärt die vielen Rupturen vor der Einführung der Sänger'schen Methode - aber auch diese ist: nach Everke ${ }^{1}$ ) noch nicht sicher genug, vielmehr muss man nach Everke zar Verhütung von Rupturen auch die Decidua nebst den angrenzenden Muskelschichten durch eine versenkte Naht 2 ) schliessen. Es ist mir nicht zweifelhaft, dass durch die Contraction und Retraction des puerperalen Uterus auch eine ursprünglich dicht schliessende Naht Lücken bekommen kann: Die Muskelränder weichen zurück, und es bildet sich eine Narbe, die nur vom Peritoneum und der Schleimhaut geliefert wird, also sehr dünn und zur Ruptur disponirt ist.

Diese beiden die Narbe des klassischen Kaiserschnitts schwächenden Momente kommen beim vaginalen Kaiserschnitt gar nicht oder kaum in Betracht. Die durchschnittenen Uteruspartien sind nach der Entleerung des Uterus nicht dicker als $1 \mathrm{~cm}$ und lassen sich daher sehr bequem und sicher durch durchgreifende Nähte, welche in Abständen von $1 \mathrm{~cm}$ angelegt werden, vereinigen. Fine puerperale Umformung des Nahtgebietes, durch welche es zu Lücken in der Wunde kommen kann, findet kaum statt, da man ja den vaginalen Kaiserschnitt bei unentfaltetem Collum ausführt, welches dann im Wochenbett nicht die Rückbildungsprocesse durchzumachen hat, wie ein gedehntes and entfaltetes Collum. Allerdings ist es, wenn man sich die Vortheile einer leicht anzulegenden und auch in der Folgezeit die Wundränder aneinander baltenden Naht siehern will, nöthig, die Spaltung möglichst wenig über den inneren Muttermund hinauf $z$ u fïhren and $z$ a diesem Behufe die von mir als das Normalverfahren empfohlene Spaltung beider Uteruswände zu machen!

ad 2. Die septische Infection wird eine Wundheilung nach vaginalem Kaisersehnitt viel weniger stören, als vach klassischem Kaisersehnitt, weil in dor Drainage des ante- und retrocervicalen Raumes ein Mittel vorhanden ist, die Wundsecrete nach aussen zu leiten.

ad 3. Dass die Placentarinsertion in die Nähe der Narben eines vaginalen Kaiserschnitts geräth, ist viel seltener, als das

1) Monatssohr. f. Geburtsh. u. Gyn. Bd. 14. S. 644.

2) Siehe anch v. Fellenberg, Zur Narbendehiscenz in der Wand des graviden Uterus nach früberen gynäkologischen Operationen. Dieses Archiv. Bd. 71. H. 2. 
gleiche Ereigniss beim klassischen Kaiserschnitt, da es sich im ersteren Fall um eine Placenta praevia handeln muss.

ad 4. Eine Ueberdehnung des Uterus durch Zwillinge ${ }^{1}$ ) oder Hydramnion trifft die Narbe eines klassischen Kaiserschnitts viel mehr als die kaum über den inneren Muttermund hinauf ragenden Narben des vaginalen Kaiserschnitts.

Man sorge also - wie man das leicht kann - beim vaginalen Kaiserschnitt für eine exacte Vereinigung der durchschnittenen vorderen und hinteren Uteruswand, so wird man Narben schaffen, welche bei einer späteren Geburt trotz ihrer Dehnung bei der Bildung des Durchtrittsschlauchs nicht reissen.

Noch weniger ist es angängig, wie Labhardt es thut, die Narbenzerreissungen nach Uterusrupturen im unteren Gebärmutterabschnitt gegen den vaginalen Kaiserschnitt auszuspielen. Denn diese Narben sind, wenn die frische Ruptur nicht genäht war wie das naturgemäss in keinem der Peham'schen ${ }^{2}$ ), von Labhardt angezogenen Fâlle geschehen war - sehr wenig resistent. $\mathrm{Ja}$, in einem Falle, welchen Breusis) beobachtete, war von vornherein nach der Heilung der Ruptur eine Spalte vorhanden, durch welche der Finger in eine im Ligament gelegene Höhle eindrang. Aber auch wenn incomplete Uterusrupturen genäht werden, so geschieht das häufig unvollkommen, weil das obere Ende des Risses mit der Naht nicht erreicht werden kann oder sogar absichtlich, zwecks Drainage der Ligamenthöhle, nicht vernäht wird.

So wird es sich wohl auch in dem Fall von Labhardt verhalten haben, der ganz zu Unrecht als „Ruptur in der Narbe einer Dührssen'schen tiefen Cervixincision" bezeichnet wird. Ich habe niemals bei Mehrgebärenden mit erhaltenem Collum eine vordere, tiefe, bis zum inneren Muttermund reichende Cervixincision ohne besondere Eröffnung des Schoidengewölbes, also ohne vaginalen Kaiserschnitt, ausgeführt oder empfohlen, und protestire, ebenso wie den Hofmeier'schen Fällen gegenüber ${ }^{4}$ ), dagegen, dass das Konto der tiefen Cervixincisionen — die ich nur für Fälle (meistens Erstgebärende) mit entfalteter supravaginaler Cervixpartie empfohlen habe - mit derartigen Fällen belastet wird.

1) Siehe den Fall von Wayer, M. f. Geb. u, Gyn. Bd. 6. H. 2.

2) O. f. Gyn. 1902. No. 4.

3) Wiener med. Blätter. 1883. 24, Juni.

4) Dührssen, C. f. G. 1904. No. 13. 
Auch die von Labhardt angezogenen Rupturen von Cervixrissnarben in Fällen, wo die frischen Cervixrisse symptomlos verliefen, sprechen wohl, wie er auch herrorhebt, gegen die Bossi'sche Methode ohne nachfolgende Nahtvereinigung der entstandenen Risse, aber nicht gegen, sondern für den vaginalen Kaiserschnitt mit seiner exacten Nahtvereinigung der gesetzten Wunden.

Das "prudenter agas et respice finem", welches Labhardt den Anhängern eines activen Vorgehens vorhält, sollen sich diejenigen zu Herzen nehmen, welche durch forcirte Extractionen bei mangelhaft erweitertem Collum (cf. den Fall 1 von Labhardt) uncontrolirbare und nicht exact zu vernähende Risse der supravaginalen Collumpartie machen - Risse, welche bei Innehaltung der von mir für die tiefen Cervixincisionen und den vaginalen Kaiserschnitt gegebenen Vorschriften gänzlich vermieden werden. In dem Fall 1 von Labhardt hätto ich übrigens überhaupt keine Incision, sondern die Metreuryse ${ }^{1}$ ) oder die combinirte Wendung allein gemacht. -

Die Metreuryse bringt mich auf das Kapitel der künstlichen Frübgeburt, da auch für diese die Bossi'sche Methode von $\mathrm{I}_{\lrcorner} \mathrm{e}$ pold empfohlen wird. Aber auch bei der künstlichen Frühgeburt ist das Bossi'sche Instrument für die Dilatation der Cervix zum Zweck der späteren Einlegung eines Metreurynters unnöthig. Ist der Cervicalkanal für einen Finger durchgängig, so lässt sich ein dürnwandiger Braun'scher Kolpeurynter in den Uterus einführen, und ist er in seltenen Fällen nieht durehgängig, so lässt er sich schonender durch Einlegung eines Laminariastifts (cf. meinen Fall III), oder Dilatation mit Hegar'sehen Dilatatoren (of. meinen Fall I) erweitern. Denn selbst die geringe Dilatation mit dem Bossi- oder einem ähnlichen Dilatator, wie sie in der Dresdener Klinik bei der künstlichen Frühgeburt angewandt wurde, erzengte nach dem Bericht von Heller ${ }^{2}$ ) in einer Anzahl von Fällen Collumrisse.

1) Siehe Dührssen, Ueber die Bedeutung der mechanischen Dilatation des Muttermundes in der Geburtshülfe. V.d. D. Ges. f. Gyn. 1893; Ueber die Behandlung der Placenta praevia mit intrateriner Kolpeuryse. D.m. W. 1894. No. 19.

2) Uober künstliche Frühgeburt bei engem Becken mittelst des Bossischen Dilatationsverfahrens und daran sich anschliessender Metreuryse. Dieses Archit. Bd. 73. H. 3. 
Es erscheint mir bei dieser Gelegenheit zweckmässig, auf Grund meiner 15 jährigen Erfahrung meine Ansichten über die beste Art der Metreuryse kurz mitzutheilen. Am sichersten und schnellsten wirkt der Ballon von Champetier de Ribes, welchen ich in der von Müller angegebenen Modification verwende. Ist die sofortige Entbindung nöthig, so wird der Ballon mit der Hand durchgezogen. Gelingt die schnelle Extraction nicht, so wird an dem Ballon ein selbstthätiger Zug angebracht, der gewöhnlich in wenigen Stunden den Ballon zur Ausstossung bringt. Ist keine solche Eile nöthig, so kann man zunächst von jedem Zug absehen und diesen nur dann anbringen, wenn eine nach einigen Stunden kräftiger Wehenthätigkeit vorgenommene Untersuchung ergiebt, dass die Erweiterung des Muttermundes keine besonderen Fortschritte gemacht hat. Hat der Ballon den Durchtrittsschlauch passirt, so ist ein Hinderniss für die schonende Entwickelung des Kindes seitens der weichen Geburtswege nicht mehr vorhanden. Man warte aber nach der spontanen Ausstossung dieses Ballons die Geburt ruhig ab, wenn keine Gefahr seitens der Mutter oder des Kindes vorhanden ist.

Man wende überall diesen Ballon an, wo er eingeführt werden kann. Die Einführung ist bei nur für einen Finger durchgängigem Collum schwierig und schmerzhaft. In solchen Fällen führe ich zunächst einen dünnwandigen Braun'schen Kolpeurynter ein. Was dessen Wirkung anbelangt, so ist sie bezüglich einer baldigen Wehenerzeugung nur sicher, falls man an dem Kolpeurynter einen constanten selbstthätigen Zug anbringt, wie ich ${ }^{1}$ ) ihn zuerst empfohlen habe. Ich bringe diesen Zug auch heute noch in derselben einfachen Art meines ursprünglichen Vorschlags an, indem ich um das Fussende des Betts eine Schlinge lege, um diese Schlinge den Kolpeurynterschlauch herumführe, denselben so stark anziehe, als es die Schwangere ohne Schmerzen aushalten kann, und nun die beiden Schenkel des Kolpeurynterschlauchs zusammenbinde oder zusammenklemme.

Lassen die Wehen nach, so findet man in der Regel den Kolpeurynter in die Scheide ausgestossen. Nur in den Fällen, wo man den Kolpeurynter sehr prall gefüllt hat, kann der Wehennachlass hierdurch bedingt sein, so dass man ihn bis auf $300 \mathrm{ccm}$ entleeren muss.

1). Verh. d. deutschen Gesellsch. für Gyn. zu Breslau. 1893. 
Ist der Braun'sche Kolpeurynter spontan in die Scheide ausgestossen, so ist es nicht immer möglich, sofort die Entbindung anzuschliessen, da dieser Ballon, auch ohne dass die Cervix sich völlig erweitert, in die Scheide geboren werden kann.

In Fällen also, in denen grössere Eile nöthig ist und in denen zunächst die Einführung des Müller'schen Ballons unthunlich war, ist es zweekmässig, nach einigen Stunden den Braun'schen Kolpeurynter durch den Müller'schen Ballon zu ersetzen.

Ich bin der festen Ueberzengung, dass der Praktiker bei Eklampsie bessere Resultate haben wird, wenn er von den neueren Methoden die Metreuryse verwendet und in schweren Fällen einen Specialisten zuzieht, als wenn er selbst jeden Fall mit Hülfe der Bossi'schen Methode za Ende zu bringen sucht.

Zum Schluss möchte ich betonen, dass ich auf dem hier bẹsprochenen Gebiet dasselbe Ziel verfolge wie Leopold, unsere Differenz bezieht sich nur auf die Mittel, mit welchen wir dieses Ziel erreichen wollen. Nach den Erfahrungen, welche besonders in der geburtshülflichen Klinik der Charité mit all' den in Betracht kommenden Vethoden gesammelt sind, haben die verschiedenen von mir empfohlenen Methoden sich der Bossi'sehen Methode überlegen erwiesen. Es liegt daher eine gegründete Veranlassung dafür vor, dass diejenigen Kliniken, in welchen letatere Methode ausschliesslich angewandt wird, auch die übrigen Methoden systematisch prüfen. Ich bin überzeugt, dass das Resultat sich zu folgendem Schluss verdichten wird: Bei verstrichenem Collum ist die Bossi'sche Methode überflüssig, bei erhaltenem Collum gefährlich für Mutter und Kind! 